\title{
MultiView High Precision VLBI Astrometry at Low Frequencies
}

\author{
María J. Rioja ${ }^{1,2,3}$, Richard Dodson ${ }^{1}$, Gabor Orosz ${ }^{4}$, Hiroshi Imai ${ }^{4,5}$, and Sandor Frey ${ }^{6}$ \\ ${ }^{1}$ ICRAR, M468, University of Western Australia, 35 Stirling Highway, Perth 6009, Australia; maria.rioja@icrar.org \\ ${ }^{2}$ CSIRO Astronomy and Space Science, 26 Dick Perry Avenue, Kensington WA 6151, Australia \\ ${ }^{3}$ OAN (IGN), Alfonso XII, 3 y 5, E-28014 Madrid, Spain \\ ${ }^{4}$ Graduate School of Science and Engineering, Kagoshima University, 1-21-35 Korimoto, Kagoshima 890-0065, Japan \\ ${ }^{5}$ Science and Engineering Area of Research and Education Assembly, \\ Kagoshima University, 1-21-35 Korimoto, Kagoshima 890-0065, Japan \\ ${ }^{6}$ Konkoly Observatory, MTA Research Centre for Astronomy and Earth Sciences, Konkoly Thege Miklósút 15-17, H-1121 Budapest, Hungary \\ Received 2016 November 4; revised 2016 December 20; accepted 2017 January 4; published 2017 February 9
}

\begin{abstract}
The arrival of the Square Kilometer Array (SKA) will revitalize all aspects of Very Long Baseline Interferometry (VLBI) astronomy at lower frequencies. In the last decade, there have been huge strides toward routinely achieving high precision VLBI astrometry at frequencies dominated by tropospheric contributions, most notably at $22 \mathrm{GHz}$, using advanced phase-referencing techniques. Nevertheless, to increase the capability for high precision astrometric measurements at low radio frequencies $(<8 \mathrm{GHz})$, an effective calibration strategy of the systematic ionospheric propagation effects that is widely applicable is required. Observations at low frequencies are dominated by distinct direction-dependent ionospheric propagation errors, which place a very tight limit on the angular separation of a suitable phase-referencing calibrator. The MultiView technique holds the key to compensating for atmospheric spatial-structure errors, by using observations of multiple calibrators and twodimensional interpolation in the visibility domain. In this paper we present the first demonstration of the power of MultiView using three calibrators, several degrees from the target, along with a comparative study of the astrometric accuracy between MultiView and phase-referencing techniques. MultiView calibration provides an order of magnitude improvement in astrometry with respect to conventional phase referencing, achieving $\sim 100 \mu$ as astrometry errors in a single epoch of observations, effectively reaching the thermal noise limit. MultiView will achieve its full potential with the enhanced sensitivity and multibeam capabilities of SKA and the pathfinders, which will enable simultaneous observations of the target and calibrators. Our demonstration indicates that the $10 \mu$ as goal of astrometry at $\sim 1.6 \mathrm{GHz}$ using VLBI with SKA is feasible using the MultiView technique.
\end{abstract}

Key words: astrometry - quasars: general - stars: individual (OH128.6-50.1, WX Psc) techniques: high angular resolution - techniques: interferometric

\section{Introduction}

Very Long Baseline Interferometry (VLBI) observations hold the potential to achieve the highest astrometric accuracy in astronomy, provided that the fringe phase observable can be calibrated (Alef 1988). The development of advanced phasereferencing (PR) techniques to compensate for tropospheric propagation errors have led to routinely achieving microarcsecond ( $\mu$ as) astrometry at frequencies between $\sim 10$ and a few tens of $\mathrm{GHz}$ using alternating observations of the target and a nearby calibrator, which can be up to a few degrees away (Reid \& Brunthaler 2004; Honma et al. 2008). The increasingly fast tropospheric fluctuations at higher frequencies set an upper threshold for application of PR techniques at $\sim 43 \mathrm{GHz}$ (with only a single case at $86 \mathrm{GHz}$; Porcas \& Rioja 2002). More recently, the development of phase-calibration techniques using (nearly) simultaneous observations at multiple millimeter wavelengths, that is, Source Frequency Phase Referencing (Rioja \& Dodson 2011) and Multi Frequency Phase Referencing (Dodson et al. 2016), have extended the capability to perform $\mu$ as astrometry up to millimeter wavelengths. This capability for accurate astrometry has resulted in wide applicability to many scientific problems (Reid \& Honma 2014 and references therein). Nevertheless, the application of these advanced PR techniques to relatively low frequencies $\leqslant 8 \mathrm{GHz}$ is hindered by the contribution of ionospheric propagation effects, increasingly dominant at lower frequencies, which have a different nature from tropospheric effects. The unpredictability of the spatial irregularities in the plasma density in the ionosphere introduces differential path variations between the sky directions of the two sources, and these propagate into the systematic position errors even for small source separations. In addition, at the lowest frequencies, temporal variations are also an issue. These are both responsible for degrading the positional accuracy achieved with this technique and, eventually, preventing the phase connection process and the use of conventional phase referencing.

Therefore, a new strategy is required to overcome the limitations imposed by the ionospheric propagation medium and to reach the full potential of the instruments working in these spectral regimes, such as the Square Kilometer Array (SKA) that will have VLBI capability between 0.3 and $14 \mathrm{GHz}$. In general, observations that involve more than one calibrator have demonstrated advantages for astrometric VLBI at low frequencies (Fomalont \& Kopeikin 2002; Rioja et al. 2002; Doi et al. 2006). The alternative is the unusual configuration when a target and a strong calibrator lie within the field of view of the VLBI antennas (i.e., an "in-beam" calibrator), and thus can be observed simultaneously (Marcaide \& Shapiro 1984; Fomalont et al. 1999; Rioja \& Porcas 2000; Wrobel et al. 2000). The results obtained with this approach are positive; however, its widespread application is still limited by sensitivity. Another useful approach is when there are two calibrators aligned with, but on opposite sides of, the target (Fomalont \& 
Table 1

All Observed Sources: Four Quasars and One OH Maser Line Source (also known as WX Psc)

\begin{tabular}{|c|c|c|c|c|c|c|}
\hline Source Name & Alias & $\begin{array}{c}\text { R.A. } \\
(\mathrm{h} \mathrm{m} \mathrm{s})\end{array}$ & $\begin{array}{l}\text { Decl. } \\
\left({ }^{\circ}, \prime^{\prime \prime}\right)\end{array}$ & $\begin{array}{l}\sigma_{\text {R.A. }} \\
\text { (mas) }\end{array}$ & $\begin{array}{l}\sigma_{\text {decl. }} \\
\text { (mas) }\end{array}$ & $\begin{array}{c}S_{1.6 \mathrm{GHz}} \\
\left(\mathrm{Jy} \mathrm{beam}^{-1}\right)\end{array}$ \\
\hline $\mathrm{J} 0121+1149$ & $\mathrm{C} 1$ & 012141.595044 & +114950.41304 & 0.10 & 0.10 & 2.1 \\
\hline $\mathrm{J} 0113+1324$ & $\mathrm{C} 2$ & 011354.510365 & +132452.47783 & 0.26 & 0.38 & 0.08 \\
\hline $\mathrm{J} 0106+1300$ & $\mathrm{C} 4$ & 010633.356509 & +130002.60390 & 0.14 & 0.19 & 0.07 \\
\hline OH128.6-50.1 & $\mathrm{OH}$ & 010625.98 & +123553.0 & $\ldots$ & $\ldots$ & 0.11 \\
\hline
\end{tabular}

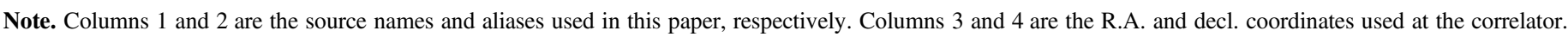

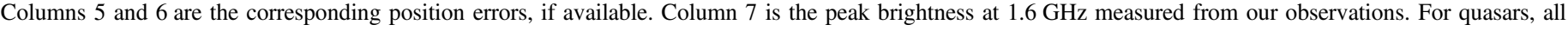

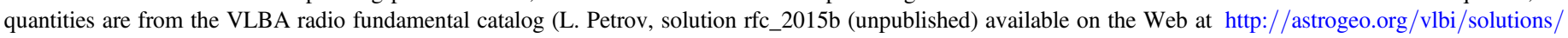
rfc_2015b). For the OH maser, the position comes from the SIMBAD astronomical database (http://adsabs.harvard.edu/abs/2000A\%26AS..143.... 9 W).

Kopeikin 2002). During observations, the telescopes alternate every few minutes between the three sources, and in the analysis, successive scans on the calibrators are used for the spatial and temporal interpolation to the enclosed position and scan time of the target source. The rare source configuration required for this approach to work results in limited applicability, and the calibration time overhead is large.

In this paper we present results from the MultiView technique which, by deriving two-dimensional (2D) phase screens from observations of three or more calibrators, achieves a superior mitigation of atmospheric errors that results in increased precision astrometry, along with wide applicability by relaxing the constraints on the angular separation up to a few degrees, and does not require alignment of sources. The scope of application is the low frequency regime, where the performance of PR is degraded due to the spatial structure of the dominant ionospheric errors. It is a development of the "cluster-cluster" VLBI technique, which allowed simultaneous observations of a target and multiple calibrators around it by replacing single telescopes by sites with multiple elements (Rioja et al. 1997). The ability of the "cluster-cluster" technique to address the ionospheric effects has been demonstrated with joint observations between connected interferometer arrays at $1.6 \mathrm{GHz}$ of a target and three calibrator sources (Rioja et al. 2002, 2009). Despite these benefits, its use has been limited by the shortage in compatible observing sites and the complexity in its implementation.

We revisit this technique in light of the next generation of instruments for low frequency observations that will become operational over the course of the next decade, such as the Australian SKA Pathfinder (ASKAP) in the near future, and SKA in the longer term. These have multibeam capability as a built-in feature. We believe that the implementation of MultiView techniques will enhance the performance of VLBI observations, by providing higher precision astrometric measurements of many targets at low frequencies.

In this paper, we describe the observations in Section 2; the basis of the MultiView technique in Section 3; Section 4 presents a demonstration and quantifies its astrometric capabilities, along with a comparative study with outcomes from conventional PR techniques for a range of targetcalibrator angular separations, including in-beam phase referencing, using VLBA observations at $1.6 \mathrm{GHz}$; and Section 5 contains discussions and conclusions.

\section{Observations}

In an effort to demonstrate the improvements by MultiView calibration over conventional techniques, we conducted two epochs of observations with the $\mathrm{NRAO}^{7}$ Very Long Baseline Array (VLBA) separated by one month, on 2015 June 8 (epoch I, obs. ID: BO047A7) and July 7 (epoch II, obs. ID: BO047A4), at $1.6 \mathrm{GHz}$. Both epochs of observations used identical setups with a duration of $\sim 4 \mathrm{hr}$. Table 1 lists the source names and coordinates, and Figure 1 shows the distribution in the sky.

The observations consisted of alternating scans switching between all the sources with a duty cycle of $\sim 5$ minutes. The two sources in the center of the distribution, the $\mathrm{OH}$ maser source and the quasar $\mathrm{C} 4$, were observed simultaneously because they lie within the primary beam of the VLBA antennas. They are the targets of the analyses presented in this paper, allowing the MultiView calibration to be tested for both a maser line and quasar continuum observations simultaneously. The sessions were long enough to ensure sufficient $(u$, $v$ ) coverage and sensitivity, spending $\sim 40 \%$ of the time on the $\mathrm{OH}-\mathrm{C} 4$ pair with alternating 30-90 s scans on the $\mathrm{C} 1, \mathrm{C} 2$, and C3 calibrators. Both epochs were observed at a similar time range around early morning, 12:06:00-16:06:00 UT for epoch I and 11:12:00-15:12:00 UT for epoch II, when the variations in the ionosphere and its effects on astrometry are expected to be the largest.

The two-bit quantized signals were recorded in dual circular polarization with $256 \mathrm{Mbps}$ using four intermediate frequency (IF) bands, each with a bandwidth of $8 \mathrm{MHz}$. The IFs were spread out over $300 \mathrm{MHz}$, centered around the four groundstate $\mathrm{OH}$ maser lines of $1612,1665,1667$, and $1720 \mathrm{MHz}$ and the HI line at $1420 \mathrm{MHz}$. Each band had a channel spacing of $1.95 \mathrm{kHz}$, corresponding to a velocity resolution of $0.36 \mathrm{~km} \mathrm{~s}^{-1}$. All bands were correlated in a single run, using two phase centers for the observations of the $\mathrm{OH}-\mathrm{C} 4$ pair. For the $\mathrm{OH}$ maser source, only the $1612 \mathrm{MHz}$ transition provided useful astrometric data.

\section{Astrometric Data Analyses}

We carried out a comparative astrometric study using different calibration techniques and angular separations, summarized in

\footnotetext{
The National Radio Astronomy Observatory is a facility of the National Science Foundation operated under cooperative agreement by Associated Universities, Inc.
} 


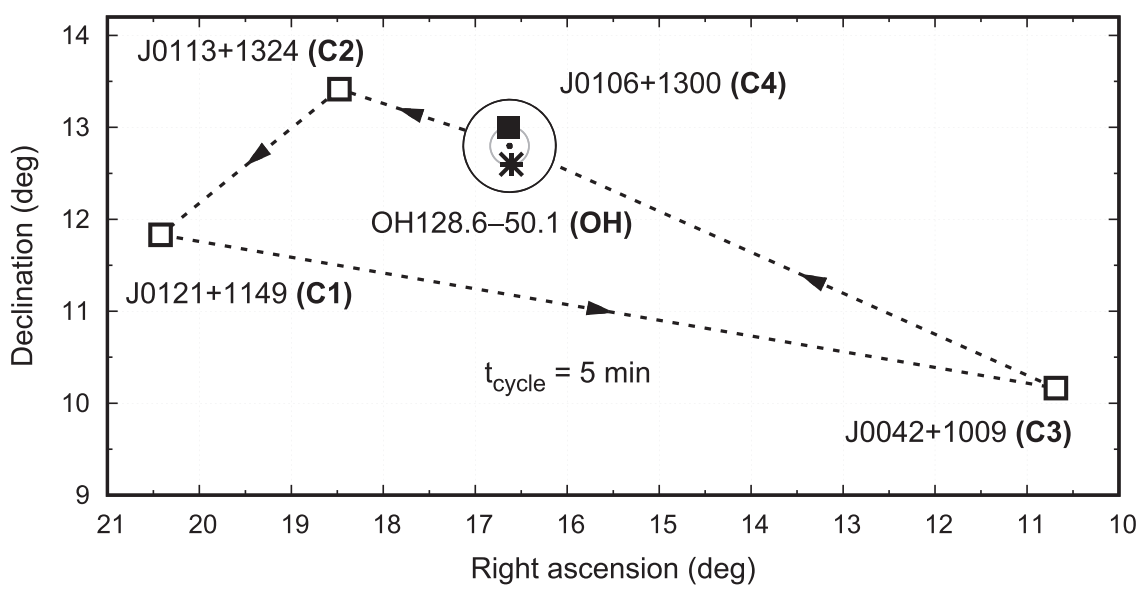

Figure 1. Sky distribution of the sources observed with the VLBA at $1.6 \mathrm{GHz}$. Table 1 lists the source coordinates. Dashed lines and arrows mark the source switching order during the observations with 5 minute duty cycles. Star and solid symbols mark the simultaneously observed OH-C4 pair, with the VLBA antennas pointed halfway between the two. The two concentric circles represent the half-power beam width and full beam width of the antennas. Both $\mathrm{OH}$ and $\mathrm{C} 4$ are targets in the astrometric analyses (see Table 2 for description of analyses). C1 was used as the fringe finder.

Table 2

Astrometric Analyses Compared in this Paper, Along with Aliases Used Throughout the Text

\begin{tabular}{lllll}
\hline \hline $\begin{array}{l}\text { Astrometric } \\
\text { Calibration }\end{array}$ & $\begin{array}{l}\text { Target } \\
\text { Source(s) }\end{array}$ & $\begin{array}{l}\text { Reference } \\
\text { Source }\end{array}$ & $\begin{array}{l}\text { Angular } \\
\text { Separation }\end{array}$ & $\begin{array}{l}\text { Analysis } \\
\text { ID }\end{array}$ \\
\hline $\begin{array}{l}\text { Single Calibrator } \\
\text { PR }\end{array}$ & $\mathrm{C} 4$ & $\mathrm{C} 2$ & 1.84 & $\mathrm{PR}_{2^{\circ}}$ \\
PR & $\mathrm{C} 4$ & $\mathrm{C} 1$ & 3.88 & $\mathrm{PR}_{4^{\circ}}$ \\
PR & $\mathrm{C} 4$ & $\mathrm{C} 3$ & 6.49 & $\mathrm{PR}_{6^{\circ}}$ \\
"in-beam" PR & OH & $\mathrm{C} 4$ & 0.4 & $\mathrm{PR}_{\text {in-beam }}$ \\
\hline Three Calibrators & & & & \\
MV & $\mathrm{C} 4$ & $\mathrm{C} 1, \mathrm{C} 2, \mathrm{C} 3$ & $1.84,3.88,6.49$ & $\mathrm{MV}_{\mathrm{QSO}}$ \\
MV & OH & $\mathrm{C} 1, \mathrm{C} 2, \mathrm{C} 3$ & $2.0,3.81,6.30$ & $\mathrm{MV}_{\mathrm{OH}}$ \\
\hline
\end{tabular}

Note. Column 1 gives the calibration techniques: using a single calibrator, regardless whether the observations of the pair are carried out using source switching or simultaneously observed, and using three calibrators; PR-phase referencing and MV-MultiView. Columns 2 and 3 list the target and reference sources, respectively. Column 4 is the target-calibrator(s) angular separation. Column 5 is the analysis identification name used throughout the text. All analyses were carried out for epochs I and II, separated by one month.

Table 2, on the same observations. The analyses comprise conventional PR techniques using pairs of sources (i.e., one calibrator and one target source) with angular separations of $\sim 0{ }^{\circ} .4$ (i.e., in-beam), $2^{\circ}, 4^{\circ}$, and $6^{\circ}$, and MultiView (MV) techniques using four sources (i.e., three calibrators and one target source). The targets are the quasar $\mathrm{C} 4$ and the $\mathrm{OH}$ maser source, while the calibrators are quasars in all cases. We use the alternating quasaronly observations for the comparison between PR with a range of angular separations and MV techniques, using $\mathrm{C} 4$ as the target; using the $\mathrm{OH}$ maser source as target, we compare in-beam PR with $\mathrm{C} 4$ as reference, $0^{\circ} 4$ away, to MV using three calibrators $2^{\circ}$, $4^{\circ}$, and $6^{\circ}$ away. Note that the MV observations in this paper involve source switching. The repeatability of the astrometric measurements between the two epochs (i.e., inter-epoch differences) is the chosen figure of merit for our comparative study of the calibration strategies. For the quasars-only analysis, the repeatabilities are a direct indication of the uncompensated ionospheric errors remaining after calibration, given that no source position changes are expected. For the $\mathrm{OH}$ maser analysis, the apparent source motion due to the proper motion and parallax
Table 3

Weights Used to Implement the Direction-dependent Ionospheric Calibration Along the Line of the Sight of the Corresponding Target Source in the MultiView Analyses

\begin{tabular}{lcc}
\hline \hline Target Source & Weight $_{1}$ & Weight $_{2}$ \\
\hline J0106+1300 (C4) & 1.147 & 0.174 \\
OH128.6-50.1 (OH) & 0.985 & 0.248 \\
\hline
\end{tabular}

Note. Column 1 lists the target sources for analyses $\mathrm{MV}_{\mathrm{QSO}}$ and $\mathrm{MV}_{\mathrm{OH}}$, respectively. Columns 2 and 3 are the weights that were applied for the bilinear interpolation between C2 and C1 ("Weight"), and between C3 and this combination ("Weight ${ }_{2}$ "), for the calibration of the corresponding target source.

needs to be accounted for first, even over a one-month timeline. Section 3.2 will cover the astrometric error analysis.

The PR analyses were carried out following standard procedures in the Astronomical Image Processing System (AIPS) software (Greisen 2003). The MV analyses were carried out following the same procedures in AIPS but with additional steps to incorporate direction-dependent effects, as described in Section 3.1. Also, we have made self-calibration maps of all sources using conventional hybrid mapping techniques in VLBI using AIPS and Difmap (Shepherd et al. 1994); these have been used to remove the source structure contribution, to provide reference points for the astrometric analysis, and to assess the quality of the calibration strategies using the fractional flux recovery (FFR) quantity (see Section 3.2).

\subsection{Basis of MultiView Direction-dependent Phase Calibration}

The MV calibration strategy corrects for the directiondependent nature of the ionospheric phase errors by using simultaneous or near-simultaneous observations of multiple calibrators around the target. Then we use a two-dimensional (2D) interpolation (hereafter bilinear) of the antenna phases, estimated along the directions of all calibrators, to provide corrections along the line of sight of the target observations. This is realized by a weighted average of the complex antenna gains, listed in Table 3, representing the relative source distribution in the sky, as shown in Figure 1 for the case of interest to this paper. This is equivalent to the treatment of the propagation medium as a wedge-like spatial structure, up to 
several degrees in size, above each antenna (Fomalont \& Kopeikin 2002; Rioja et al. 2002). The temporal structure of the propagation medium effects is best calibrated using simultaneous observations of the calibrators and the target sources in MV observations. However, when this observing configuration is not possible, one can use alternating observations of the sources, as long as the duty cycle is less than the atmospheric coherence time.

Therefore, in MV, the target position is tied to the assumed positions of the multiple calibrators weighted as in the analysis, that is, to a virtual point in the sky whose location depends on the source distribution in the sky. Instead, in PR, the measured target positions are tied to the assumed position of the corresponding (single) calibrator. Nevertheless, as long as the calibrator sources provide good fiducial points (i.e., are stationary), this virtual point is also stationary and changes between the astrometric measurements at different epochs trace the motion of the target in both MV and PR.

Our implementation of the MV direction-dependent calibration strategy is more complicated than a basic bilinear interpolation. It includes a correction for the untracked $2 \pi$ ambiguities inherent in the measured calibrator phases, which, if present, would lead to errors in the interpolated phases. Those are automatically detected by running an ambiguity check in the formation of each interpolation, using a scheme developed in house, adapting the concepts described in MartíVidal et al. (2008) to the two-dimensional interpolation case. Additionally we have the ability to steer the corrections by forcing the addition of ambiguities at the outset.

\subsection{Error Analysis}

We describe here the approaches used to quantify the uncertainties of the astrometric measurements in this paper.

(1) Repeatability errors. The changes between the measured positions at the two epochs for each analysis are indicative of uncompensated systematic ionospheric residual errors remaining after the corresponding calibration. We have used the repeatability of the measured positions between the two epochs of observations, which provide independent measurements of the relative source position, as an empirical estimate of the astrometric errors. The repeatability errors are calculated as $\sigma_{\text {pos,rep }}=\Delta_{I-I I} / \sqrt{2} * \sqrt{2 / \pi}$, where $\Delta_{I-I I}$ is the change between positions measured at epochs I and II, and $\sqrt{2 / \pi}$ is a factor that corrects for the bias introduced by having only two measurements (GraphPad 2014). These errors are a measure of the precision of the calibration method, in the absence of inherent source position changes, and we use them as the figure of merit for the comparative study. Note that while there is a limited sample of two epochs, the different analyses are carried out on the same observations, enabling a direct comparison of the compensation efficiency of the systematic errors under the same weather conditions.

(2) Thermal noise (and other random) errors. The ratio of the synthesized beam size $\left(\theta_{B}\right)$ and the signal-to-noise ratio $(\mathrm{S} / \mathrm{N})$ in the astrometric maps (i.e., PRed and MVed maps) gives an estimate of the uncertainty in the measurement of the position of a feature in the maps due to random noise, as $\sigma_{\text {pos, thermal }} \sim 0.5 \theta_{B} / \mathrm{S} / \mathrm{N}$. This error has a contribution from thermal (usually dominated by the receiver) random noise, and from residual atmospheric phase fluctuations. The latter depends on the duty cycle during the observations, the angular separation between sources, and the weather conditions. It is commonly referred to as the thermal noise error and represents the ultimate astrometric precision achievable, in the absence of any other error contributions. It is usually overwhelmed by other systematic contributions.

(3) Fractional Flux Recovery. The FFR quantity is defined as the ratio between the peak brightness in the astrometric maps (i.e., PRed and MVed maps) and the self-calibrated maps of the same source. It is a useful quantity for comparison between methods and is related to the thermal noise error. It provides an empirical estimate of the residual uncorrelated errors, such as atmospheric phase fluctuations, which result in image coherence losses. In general, image coherence losses arise from residual short-term phase fluctuations, hence it is expected to increase with larger duty cycles (in our case the duty cycle is $300 \mathrm{~s}$ ). The coherence losses also increase with the source's angular separation, due to residual long-term phase variations that distort the image. Nevertheless, neither of these quantities are sensitive to error processes that cause systematic position offsets, such as those expected from the spatial structure responsible for the direction-dependent nature of the ionospheric errors.

(4) Accuracy. The observed quasars were selected from a VLBI catalog with precise positions with accuracies $\sim 0.2$ mas (rfc_2015b). Differences of no more than a few milliarcseconds between the catalog positions and the relative astrometric measurements presented in this paper are expected, arising from the effects that effectively change the measured positions. These are the use of group delays observable in geodetic analysis compared to the phase delays in our relative astrometry analysis (Porcas 2009), expected position changes in the observed core at different frequencies (i.e., the core-shift effect), and differential structure blending effects between the observing frequencies of the catalog $(8.4 \mathrm{GHz})$ and our observations $(1.6 \mathrm{GHz})$.

\section{Results}

The main goal of these observations was to demonstrate the feasibility of MV to achieve high precision astrometry at low frequencies, along with a comparative study between MV and PR. The results presented here correspond to the analysis comprising observations from the eight continental VLBA antennas. The longest baselines of SC and MK did not detect the $\mathrm{OH}$ maser emission, and so were not included.

\subsection{Calibrated Visibility Phases and Astrometric Images: MV Versus PR}

Visibility Phases. Figure 2 shows a superposition of the residual relative visibility phases of $\mathrm{C} 4$ for a representative subset of baselines, after calibration using PR (analysis id: $\mathrm{PR}_{2^{\circ}}, \mathrm{PR}_{4^{\circ}}$, and $\mathrm{PR}_{6^{\circ}}$ ) and $\mathrm{MV}$ (analysis id: $\mathrm{MV}_{\mathrm{QSO}}$ ) for the same range of target-calibrator angular separations, from epochs I (left) and II (right). The long timescale trends in PR analysis are easily appreciated: the deviations from zero are increasingly large for pairs with larger angular separation and are different in the two epochs, with epoch II being significantly better. This is indicative of systematic residual phase errors, which depend on the weather conditions. Satellite-based Global Positioning System (GPS) data ${ }^{8}$ are consistent with epoch II ionospheric conditions being more

\footnotetext{
8 The GPS ionospheric data comes from the US Total Electron Conten Product Archive of the National Oceanic and Atmospheric Administration (NOAA): https://www.ngdc.noaa.gov/stp/IONO/USTEC/.
} 

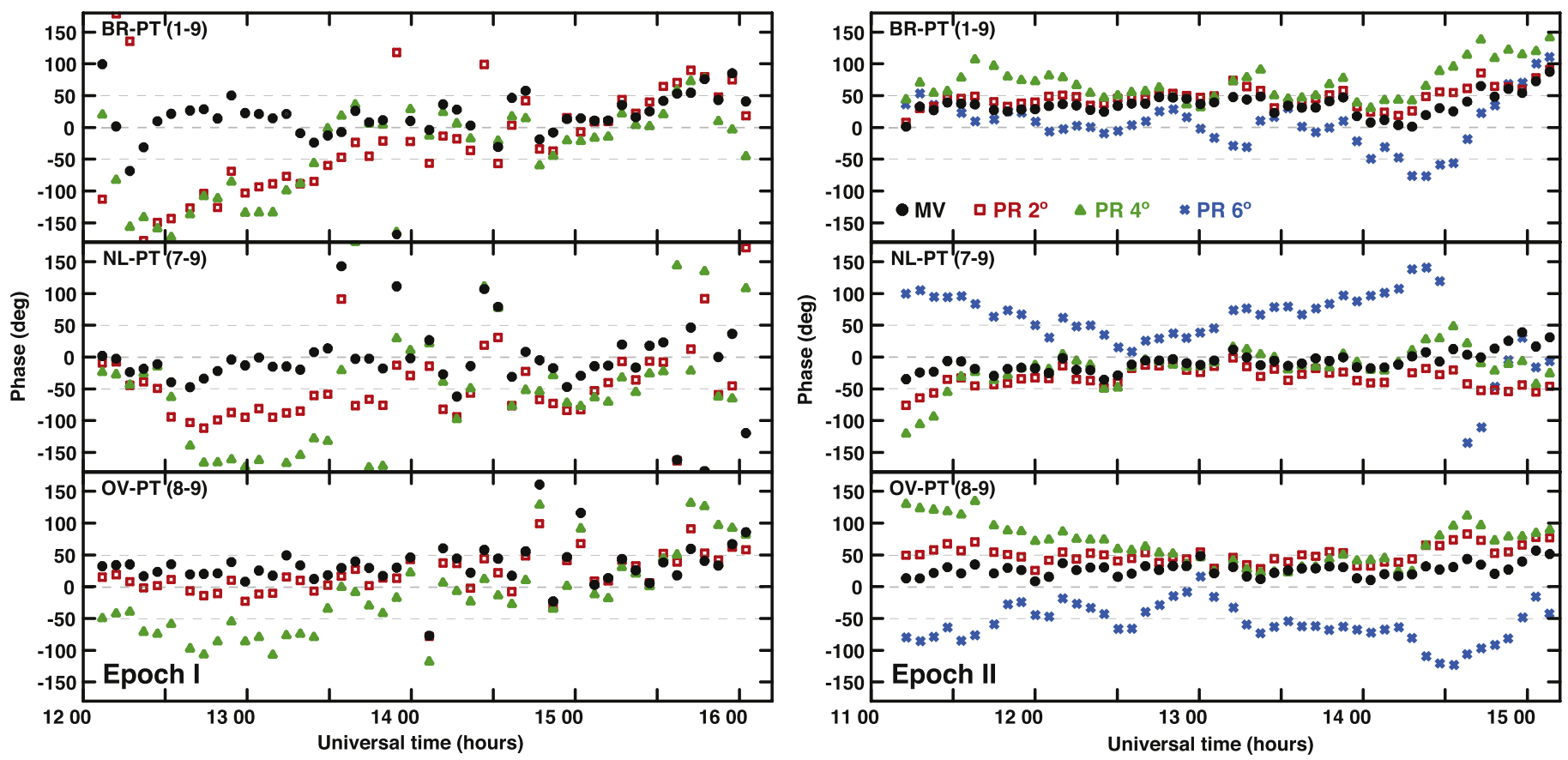

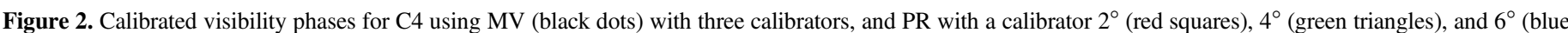

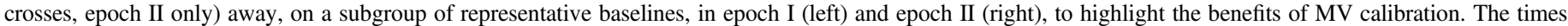

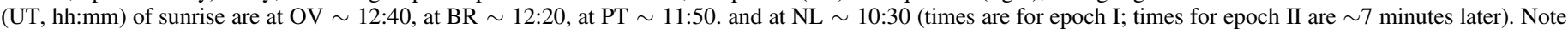
that the analysis presented in this paper was carried out using the observations with the eight continental VLBA antennas.

benign. The largest disturbances are seen at the beginning of the observations, which correspond to sunrise. Note that, in epoch II, the phases for the $6^{\circ}$ angular separation pair are larger (by about a factor of $\sim 3$ ) and with opposite sign compared to those for the $2^{\circ}$ pair and that they correspond to calibrators on opposite sides of the target. This is consistent with the expectations from a wedge-like ionospheric structure responsible for direction-dependent errors as described in Section 3.1, but does not exclude other contributions, such as instrumental effects.

Regardless of the nature of the residuals, MV results in the smallest phase residuals (significantly smaller than $\mathrm{PR}_{2}$ 。 with almost none of the signatures for sunrise or other systematic trends visible in PR, and are similar for both epochs). The same findings apply to the baselines from the eight continental VLBA antennas used in the analysis. These are indicative of superior performance in the mitigation of ionospheric errors regardless of weather conditions. This is crucial for accurate single- and multi-epoch astrometric analysis, as shown in the next section.

Astrometric Images. The calibrated visibilities are used to generate the final product of the analysis, the astrometric images, which convey the astrometric measurements presented in the next section. Figure 3 shows the MVed image for the $\mathrm{C} 4$ quasar obtained using the three calibrators together and the three PRed images using a single calibrator $\sim 2^{\circ}, 4^{\circ}$, and $6^{\circ}$ away, for the two epochs. The image degradation arises from the remaining short- and long-term residual phase variations in each analysis. A qualitative comparison suggests that the PRed images improve with closer angular separations, as expected, and that the MV is similar to $\mathrm{PR}_{2}$. at both epochs, with $\mathrm{MV}$ slightly better at epoch I, under worse weather conditions. Figure 4 shows the self-calibrated maps of the observed quasars. For a quantitative comparison, Table 4 lists the peak brightness and rms noise values in the astrometric images along with the coherence losses (i.e., FFR) estimated with respect to the self-calibrated images, for the two epochs of observations. The corresponding astrometric thermal noise errors, estimated as described in Section 3.2, are listed in Table 5. Nevertheless, as stated above, these estimates are not sensitive to residual systematic errors, which propagate into the position shifts while maintaining the quality of the image. Those are better addressed by the repeatability errors, presented in the next section.

\subsection{Astrometric Repeatability}

Astrometry is performed directly in the images of quasar C4 shown in Figure 3 by measuring the offset of the peak of brightness from the center of the map. This offset corresponds to the difference between our measurements and the catalog positions used for correlation. Figure 5(a) shows the astrometric measurements, or offsets, of the target quasar $\mathrm{C} 4$ using $\mathrm{PR}$ (analysis ID $\mathrm{PR}_{2^{\circ}}, \mathrm{PR}_{4^{\circ}}, \mathrm{PR}_{6^{\circ}}$ ) and $\mathrm{MV}$ (analysis ID: $\mathrm{MV}_{\mathrm{QSO}}$ ) at the two epochs of observations. Figure 5(b) shows an expansion of the area around the MV measurements at the two epochs. The astrometric uncertainties in the plot are the $1 \sigma$ thermal noise error bars $\left(\sigma_{\text {pos,thermal }}\right)$ listed in Table 5.

Note that, in general, while the PR and MV measurements at a given epoch are expected to differ, because they are tied to different reference points, the inter-epoch differences convey information on the source position changes (if any) in the onemonth time span between the two epochs. For stationary sources, as is the case with quasars, no or negligible position changes are expected. Therefore, one can estimate the repeatability errors using the change in the measured offsets at the two epochs for a given technique. Table 5 lists the repeatability errors, which are an empirical estimate of the astrometric precision, estimated as described in Section 3.2. It is immediately obvious that the repeatability errors are much 


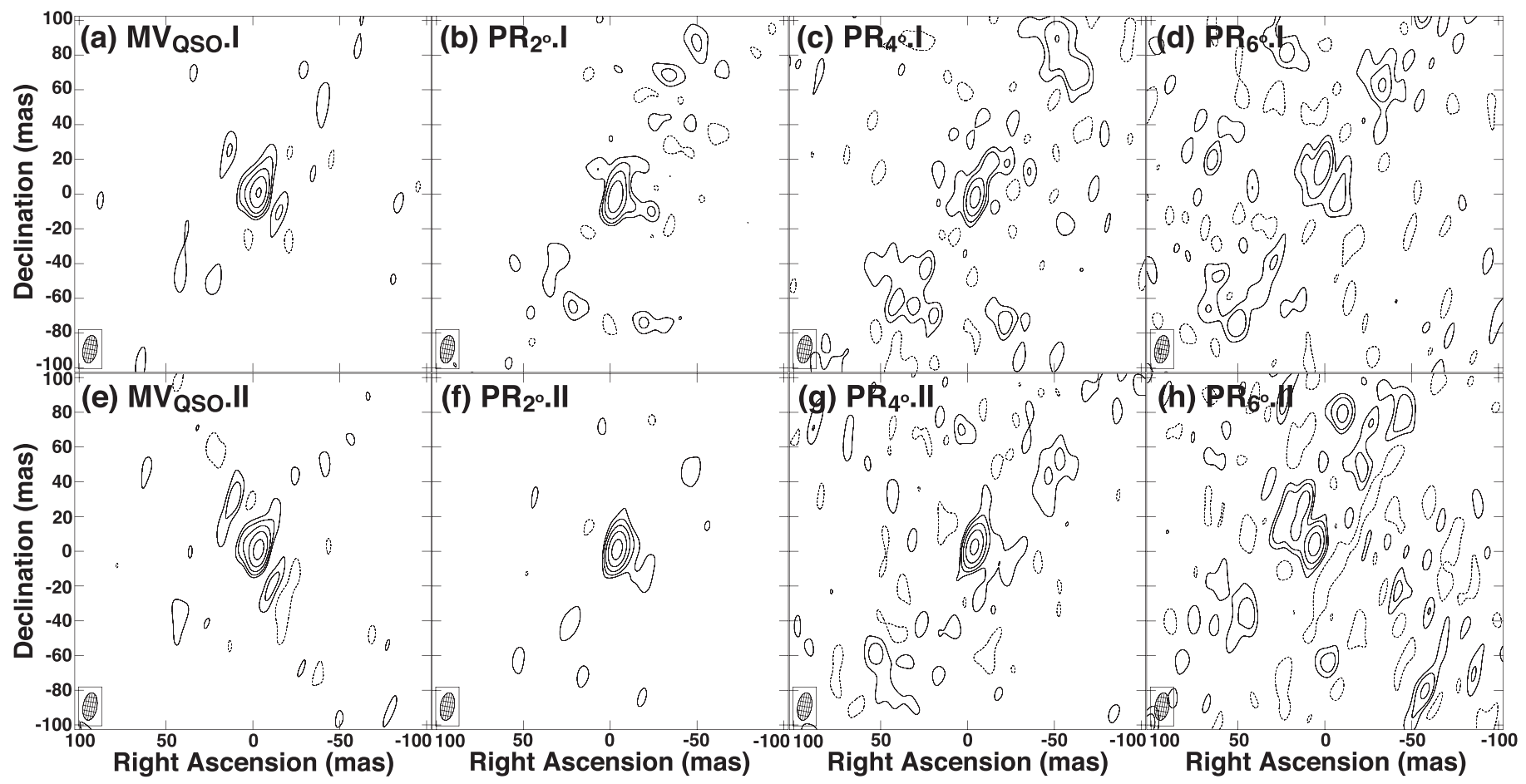

Figure 3. Astrometric images of $\mathrm{C} 4$ using analyses compared in this paper. From left to right, $\mathrm{MV}, \mathrm{PR}_{2^{\circ}}, \mathrm{PR}_{4^{\circ}}$, and $\mathrm{PR}_{6^{\circ}}$ for epoch $\mathrm{I}$ (upper row) and epoch II (lower row). The lowest intensity contour in all images are at the $3 \sigma$ level of the MV map and doubling thereafter. The images have been restored with the same beam: $8 \times 16$ mas with PA $=-10^{\circ}$. See Table 4 for values of peak brightness, rms noise, and astrometric offsets in the images.

Table 4

Outcomes from Astrometric Analyses

\begin{tabular}{|c|c|c|c|c|c|c|c|}
\hline \multirow{2}{*}{ Epoch } & \multirow{2}{*}{ Analysis ID } & \multirow{2}{*}{$\begin{array}{c}\text { Sep. } \\
\text { (degree) }\end{array}$} & \multirow{2}{*}{$\begin{array}{c}\text { Peak } \\
\text { (mJy/beam) }\end{array}$} & \multirow{2}{*}{$\begin{array}{c}\mathrm{rms} \\
(\mathrm{mJy} / \text { beam })\end{array}$} & \multirow{2}{*}{$\begin{array}{l}\text { FFR } \\
(\%)\end{array}$} & \multicolumn{2}{|c|}{ Astrometric Offset } \\
\hline & & & & & & $\overline{\Delta_{\text {R.A. }}(\mathrm{mas})}$ & $\Delta_{\text {decl. }}(\mathrm{mas})$ \\
\hline I & $\mathrm{MV}_{\mathrm{QSO}}$ & $\sim 0$ & 64 & 1 & 87 & -3.45 & 0.71 \\
\hline I & $\mathrm{PR}_{2}^{\circ}$ & 1.8 & 57 & 1 & 77 & -3.09 & -2.02 \\
\hline I & $\mathrm{PR}_{4}^{\circ}$ & 3.9 & 42 & 2 & 57 & -4.49 & -1.87 \\
\hline I & $\mathrm{PR}_{6^{\circ}}$ & 6.5 & 27 & 2 & 37 & 1.02 & 15.70 \\
\hline II & $\mathrm{MV}_{\mathrm{QSO}}$ & $\sim 0$ & 67 & 1 & 92 & -3.45 & 0.83 \\
\hline II & $\mathrm{PR}_{2^{\circ}}^{\circ}$ & 1.8 & 67 & 1 & 93 & -4.06 & 0.90 \\
\hline II & $\mathrm{PR}_{4^{\circ}}$ & 3.9 & 59 & 1 & 81 & -4.01 & 2.55 \\
\hline II & $\mathrm{PR}_{6^{\circ}}$ & 6.5 & 38 & 2 & 52 & 5.89 & 4.09 \\
\hline
\end{tabular}

Note. Column 1 is the epoch of observations. Columns 2 and 3 are the analysis ID and source angular separation, respectively. Columns 4-6 are the peak brightness, rms noise, and fractional flux recovery values measured from the astrometric images in Figure 3, respectively. Columns 7 and 8 are the astrometric offsets of the peak of brightness in the astrometric images shown in Figure 3 from the center of the map; these are shown in Figure 5(a).

larger for PR, compared to those for MV. Also, the repeatability errors are larger than the thermal noise errors for PR; instead, they are within the $1 \sigma$ thermal noise error bars for MV. The former indicates that the extended duty cycle in the observations presented in this paper, compared to that for a single calibrator, does not have a great impact on the PR astrometric errors, in agreement with expectations for error estimation in Asaki et al. (2007).

Figure 5(c) displays the repeatability errors as a function of source pair angular separations for PR analysis. This linear trend is as expected from PR analysis, as closer angular separations provide a better atmospheric compensation. The MV repeatability errors are the smallest, more than one order of magnitude smaller than those for the closest pair with PR, and are equivalent to those from a very close pair of sources (i.e., close to zero angular separation) in the PR analysis. It is worth highlighting instead that the $\mathrm{MV}$ and $\mathrm{PR}_{2}$ images are of similar quality and have similar FFR values. This underlines the insensitivity of the PRed images to large systematic errors. Also, this underlines the superior quality of the calibration of atmospheric errors using multiple calibrators, compared to that achieved with a single calibrator with the same range of angular separations, and that MV analysis leads to higher precision astrometry. This is in agreement with the findings from our previous simulation studies, where we concluded that using multiple calibrator sources with MV resulted in one order of magnitude improvement compared to PR with a single calibrator (Jimenez-Monferrer et al. 2010; Dodson et al. 2013).

Figure 5(a) also conveys qualitative information on the astrometric accuracy of the different calibration techniques since the observed quasars have well-determined positions in the rfc_2015b catalog. We expect offsets of no more than a few 
Table 5

Empirically Estimated Astrometric Errors for Measurements Presented in This Paper

\begin{tabular}{lccc}
\hline \hline \multirow{2}{*}{ Analysis ID } & \multicolumn{2}{c}{$\sigma_{\text {pos,thermal }}($ mas $)$} & \\
\cline { 2 - 3 } & Epoch I & Epoch II & $\sigma_{\text {pos,rep }}($ mas $)$ \\
\hline $\mathrm{MV}_{\text {QSO }}$ & 0.17 & 0.14 & 0.1 \\
$\mathrm{PR}_{2^{\circ}}$ & 0.19 & 0.11 & 2.7 \\
$\mathrm{PR}_{4^{\circ}}$ & 0.42 & 0.22 & 3.9 \\
$\mathrm{PR}_{6}{ }^{\circ}$ & 0.75 & 0.44 & 11.2 \\
\hline
\end{tabular}

Note. Column 1 is the calibration strategy (see Table 2 for description). Columns 2 and 3 are the thermal noise errors for epochs I and II, respectively. They have been calculated using the values listed in Table 4 with a beam of $8 \times 16$ mas PA $=-10^{\circ}$. Column 4 lists the repeatability errors, calculated from the difference between the astrometric offsets at epochs I and II listed in Table $4\left(\Delta_{I-I I}\right)$ and corrected for bias for two epochs, as $\sigma_{\text {pos,rep }}=\Delta_{I-I I} / \sqrt{2} * \sqrt{2 / \pi}$. See Section 3.2 for a description of the error analysis.

( 1-2) mas to account for differences between both measurements in all cases (see discussion in Section 3). Hence, the magnitude of each of the astrometric offsets in Figure 5(a) is indicative of the accuracy of that measurement and the method. PR offsets increase for larger source separations; MV results in the smallest offsets, which indicate higher calibration accuracy. In fact, the magnitude of the accuracies is similar to the repeatability errors listed in Table 5 .

Finally, the distribution of the measurements in Figure 5(a) is also indicative of the spatial structure of the propagation medium being a planar surface. There is a resemblance between the geometric distribution of the three PR estimates at epoch I (i.e., $\mathrm{PR}_{2^{\circ}}$.I, $\mathrm{PR}_{4^{\circ}}$.I, $\mathrm{PR}_{6^{\circ}}$.I) and epoch II (i.e., $\mathrm{PR}_{2^{\circ}}$.II, $\mathrm{PR}_{4^{\circ}}$.II, $\mathrm{PR}_{6^{\circ}}$.II). Such distributions would arise from planar spatial structures in the propagation medium above each antenna, where the size and orientation of the triangles at each epoch depend on the weather conditions at a given epoch. The triangle for epoch I is more elongated than that for epoch II. Moreover, both triangles appear rotated with respect to each other around a pivot point, which is close to the MV measurements, which remain practically unchanged (within the thermal noise error bars) at both epochs, regardless of the weather conditions, as is expected from a quality calibration.

\subsection{Astrometry on OH Maser Source: $M V$ Versus In-beam PR}

In this section, we compare the astrometric results from PR with an "in-beam" calibrator and MV with more distant calibrators, based on the analysis using the $\mathrm{OH}$ maser spectral line as the target source (analysis IDs: $\mathrm{PR}_{\text {in-beam }}$ and $\mathrm{MV}_{\mathrm{OH}}$, respectively, in Table 2). The $\mathrm{PR}_{\text {in-beam }}$ analysis uses simultaneous observations of $\mathrm{C} 4$ and the $\mathrm{OH}$ maser target source, 0.4 away; the MV analysis uses alternating observations between the target line source and the three (continuum) calibrators $\mathrm{C} 1, \mathrm{C} 2$, and $\mathrm{C} 3$, which are $\sim 2^{\circ}, 4^{\circ}$, and $6^{\circ}$ away. Figure 6(a) shows the astrometric offsets estimated at epoch II, with respect to epoch I, for both analyses. This accounts for the different reference points in PR and MV and permits a direct comparison of the inter-epoch differences. The estimated thermal noise errors are $\sigma_{\text {pos, thermal }} \sim \pm 0.5$ mas and $\sim \pm 1$ mas in right ascension and declination, respectively. Note that, in this case, the inter-epoch differences trace the expected motion of the stellar target, due to the proper motion and parallax, during the one-month interval between epochs. Hence, unlike the case of quasar-only analysis described in the section above, the astrometric changes between the two epochs are not a direct

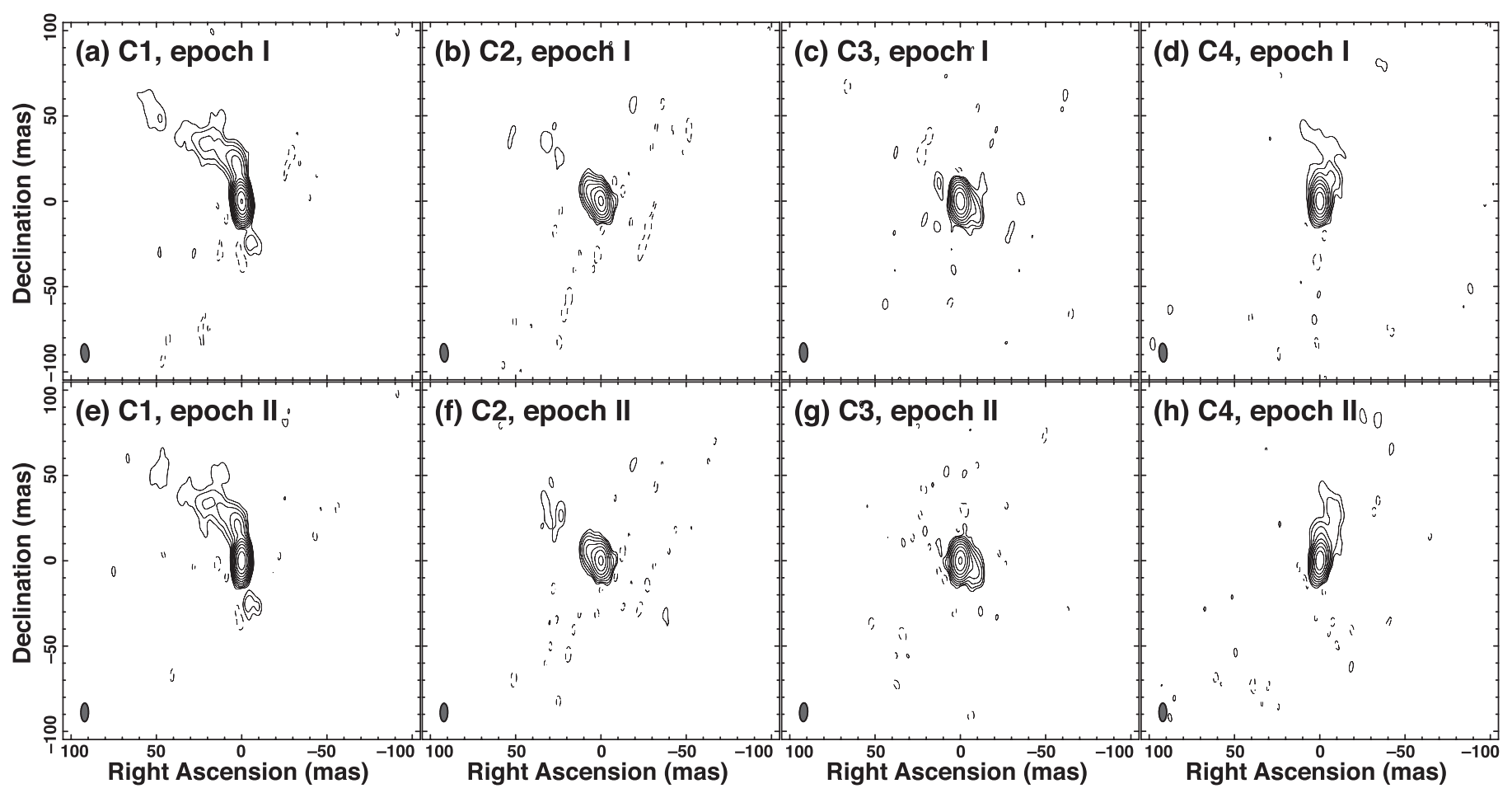

Figure 4. Self-calibrated maps of the observed quasars. The peak brightness are listed in Table 1 . The lowest intensity contour is the $3 \sigma$ level and doubling thereafter. Restoring beam is $8 \times 16$ mas with $\mathrm{PA}=-10^{\circ}$. 

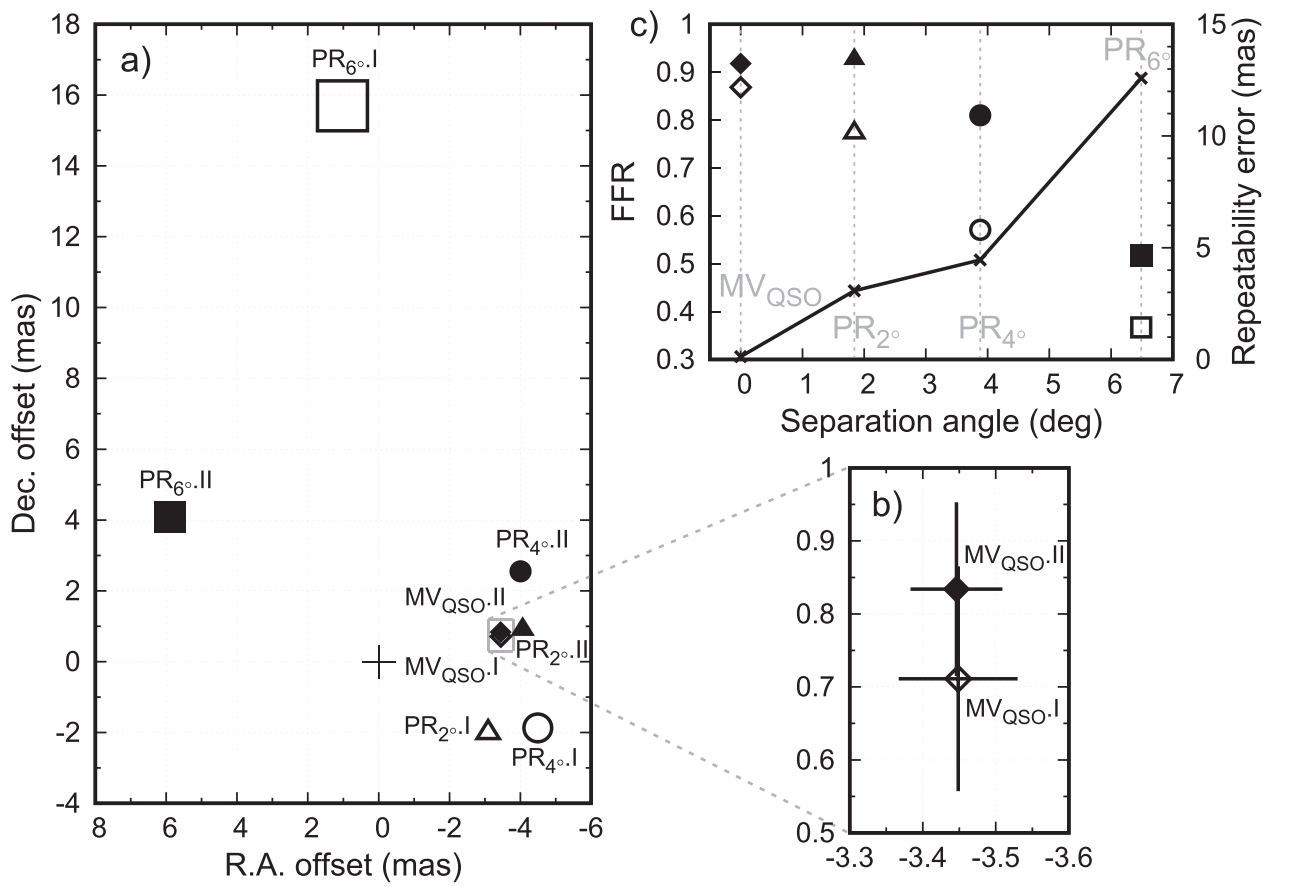

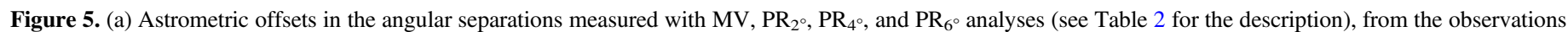

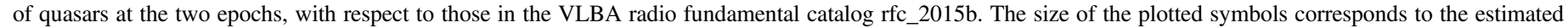

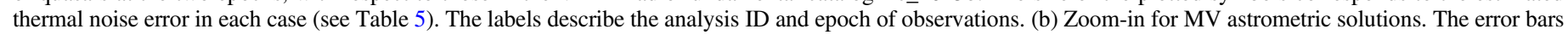

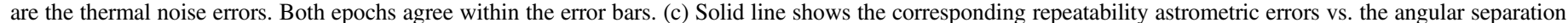

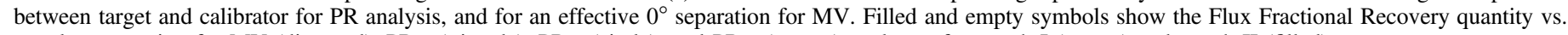
angular separation for $\mathrm{MV}$ (diamond), $\mathrm{PR}_{2^{\circ}}$ (triangle), $\mathrm{PR}_{4^{\circ}}$ (circle), and $\mathrm{PR}_{6^{\circ}}$ (square) analyses, for epoch $\mathrm{I}$ (empty) and epoch $\mathrm{II}$ (filled).
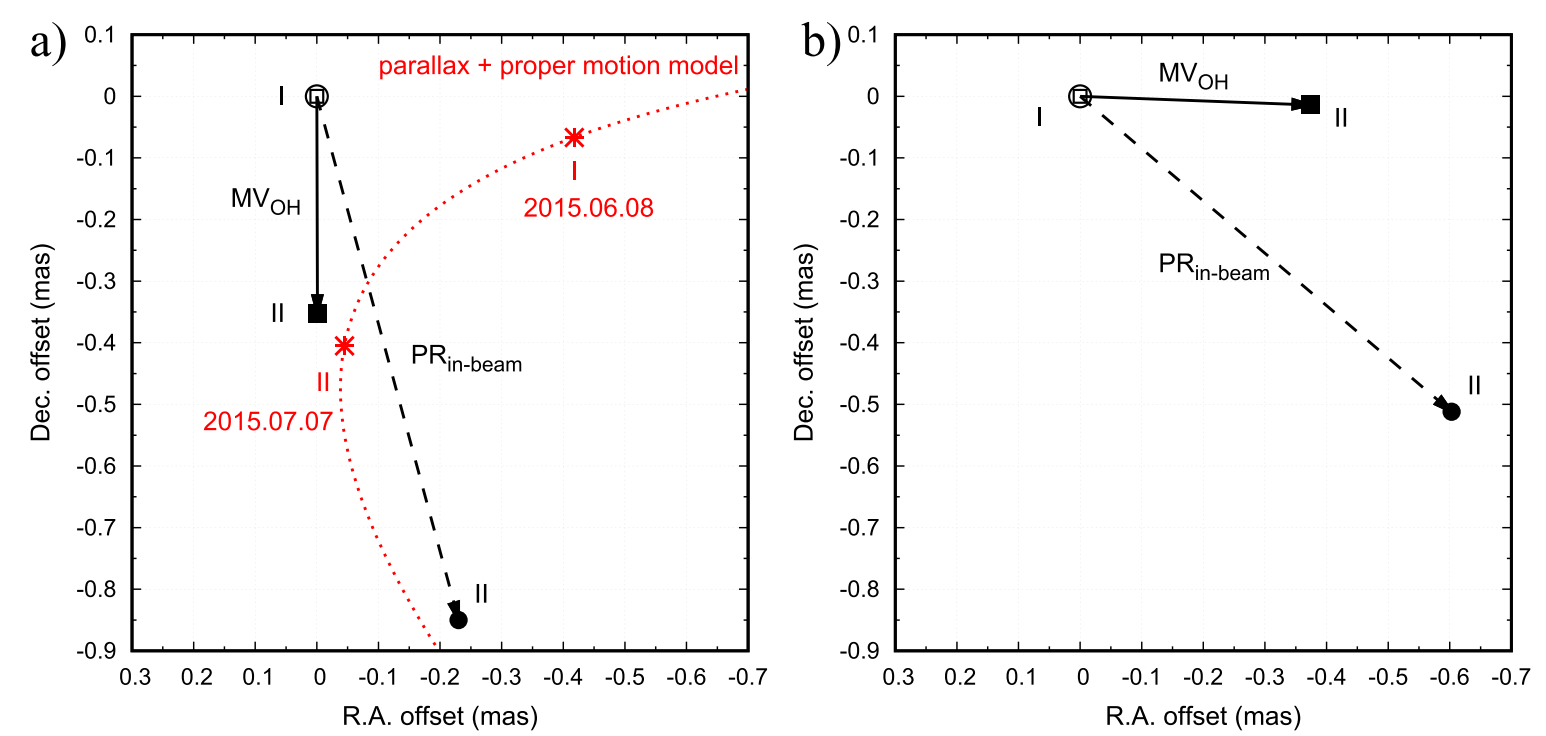

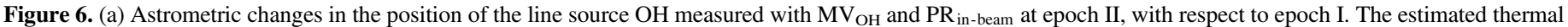

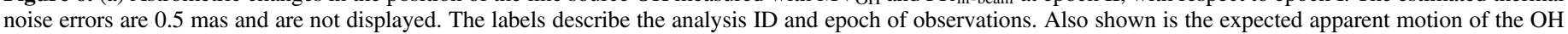

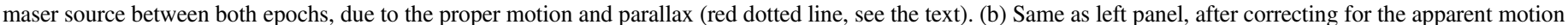
of the $\mathrm{OH}$ maser source, due to the proper motion and parallax.

measure of the repeatability errors (and the precision of the method) and an extra step is required to eliminate the contribution from the motion of the source.

We used a set of four in-beam PR observations spanning one year to measure the proper motion and parallax of the $\mathrm{OH}$ maser target source (Orosz et al. 2017). These include the two epochs described in this paper, plus two additional epochs with a similar observing configuration, except for having longer duty cycle times ( $\sim 10$ minutes), which prevented MV analysis. The measured parallax is $\pi=2.9 \pm 0.8$ mas and the proper motion is $\mu(\alpha, \quad \delta)=\left(-0.1 \pm 0.4\right.$ mas yr $^{-1}, \quad-7.5 \pm 0.7$ mas yr $\left.^{-1}\right)$. Figure 6(b) is the same as Figure 6(a), after removing the contributions from the proper motion and parallax between epochs I and II, with the repeatability errors being $\sigma_{\text {pos,rep }}^{\mathrm{MV}}=0.29$ mas for $\mathrm{MV}_{\mathrm{OH}}$ and $\sigma_{\text {pos,rep }}^{\mathrm{PR}_{\text {in }} \text {,bam }}=0.64$ mas for $\mathrm{PR}_{\text {in-beam. }}$ The repeatability errors for $\mathrm{MV}_{\mathrm{OH}}$ are half the 
magnitude for $\mathrm{PR}_{\text {in-beam }}$, albeit based on more distant calibrators. ${ }^{9}$ In this case, all measurements agree within the thermal noise errors, which are larger in the case of a weak source.

\section{Discussion and Conclusions}

Demonstration of MultiView high precision astrometry at low frequencies. The ionospheric propagation effects are the main limitation to routinely achieving high precision astrometry at frequencies $\leqslant 8 \mathrm{GHz}$, using state-of-the-art PR methods developed for higher frequencies. This is due to the distinct direction-dependent signature, which limits high precision measurements to cases when there is a suitable very close calibrator $\sim$ arcmin away. Our combination of multiple calibrators around the target results in a significant reduction of the systematic astrometric errors, from the mitigation of the spatial-structure effects, by using a bilinear interpolation to estimate the calibration along the direction of the target source.

We have presented an empirical demonstration of the superior mitigation of MV along with a comparative study with PR analysis using a single source at a range of angular separations, including "in-beam" PR. All of the analyses have been carried out using the same VLBA observations at 1.6 GHz. We have used the repeatability between two epochs of observations to provide an empirical estimate of the systematic astrometric errors, which are otherwise very difficult to quantify.

We achieve high precision MV astrometry of $\sim 100 \mu$ as in a single epoch of observations of quasars with calibrators at $2^{\circ}$ and larger angular separations, effectively reaching the thermal noise limit of the observations. This corresponds to more than an order of magnitude improvement with respect to the precision achieved using PR with a single calibrator $2^{\circ}$ away, which is $\sim 3$ mas, due to the residual systematic errors in the analysis. This underlines the importance of correcting for the spatial structure of the ionospheric residuals. The comparative improvement can be interpreted as MV compensation being equivalent to that from PR with a single calibrator $\sim 10$ times closer, in this case $\sim 0.2$ away, assuming a linear dependence between astrometric precision and target-calibrator angular separation. Also, our results indicate that there is a common limiting factor for accuracy and precision, namely, the residual ionospheric propagation errors, and both are improved by a quality calibration as provided by MV.

We have also demonstrated the performance in the weak source case using the observations of the $\mathrm{OH}$ maser line source, and compared MV using $2^{\circ}, 4^{\circ}$, and $6^{\circ}$ angular separations with "in-beam" PR using a calibrator $0^{\circ} .4$ away. The repeatability errors are larger, as expected from the lower $\mathrm{S} / \mathrm{N}$, but interestingly keep the same corresponding relative astrometric signature found in the analysis of the quasars. That is, $\mathrm{MV}_{\mathrm{OH}}$ calibration is a factor of two better, with respect to $\mathrm{PR}_{\text {in-beam, }}$ using a calibrator five times closer to the target. Therefore, we have demonstrated the benefits of using multiple calibrators, in our case with an improvement of more than one order of magnitude in astrometric precision, reaching the thermal limit of the observations, of $\sim 100 \mu$ as. In general we expect MV to be relevant for observations in the frequency

\footnotetext{
9 Due to the small number of epochs and the sub-optimal spacing, several proper motion and parallax models agree with our measured astrometry (see Orosz et al. 2017 for details). In this paper we chose the model which gave the largest repeatability errors. Using a slightly different model, the errors we measured were $\sigma_{\text {pos,rep }}^{\mathrm{MV}}=0.17$ mas and $\sigma_{\text {pos,rep }}^{\mathrm{PR}_{\text {in-beam }}}=0.44$ mas.
}

regime where the ionospheric effects continue to be the dominant source of errors, that is, in observations up to $\sim 8 \mathrm{GHz}$. We conclude that greater improvements are expected from increased sensitivity and faster duty cycles, with maximum benefits from simultaneous observations and closer source distribution, to minimize the nonlinear deviations of the actual ionospheric spatial structure above each antenna from a planar surface.

$M V$ in the context of SKA and multibeam instruments. Precise astrometric capability is of great importance in the SKA era. It is an SKA goal to achieve $10 \mu$ as astrometric accuracy at a single epoch of observations at $\sim 1.4 \mathrm{GHz}$ (Paragi et al. 2015). The high sensitivity and long baselines of SKA VLBI observations will result in a much reduced thermal noise level and high spatial resolution. Therefore, this goal is achievable as long as a sufficiently accurate ionospheric phase-calibration strategy is in place. For a single calibrator source and PR techniques, the required angular separation to the target would be $\sim 1$ arcmin (Paragi et al. 2015). This puts a very tight limit on the number of available calibrator sources, even at SKA sensitivities (Godfrey et al. 2011). This constraint on the angular separation can be significantly relaxed by using multiple calibrator sources and MV techniques, as suggested by the demonstration presented in this paper and our previous simulation studies. Additionally, the multibeam capability of SKA will allow for simultaneous observations of all sources and therefore eliminate the errors arising from short-term phase fluctuations, which result in a reduction of the coherence losses (i.e., characterized by FFR quantity) and the thermal noise errors, while improving overall performance. This applies to other instruments with multibeam systems such as ASKAP and the Westerbork Synthesis Radio Telescope with Apertif, among others. Our demonstration includes three calibratorsnote that the more calibrators used, the better, as this will allow the most accurate reconstruction of the atmospheric effects. Therefore, we expect that MV can deliver the goal of $10 \mu \mathrm{as}$ astrometry for many targets with SKA.

Next we consider other scientific applications of MV.

Near-field Cosmology. This paper used astrometric observations of a group of quasars in the role of targets and calibrators and an $\mathrm{OH}$ maser source in the ground state at $1.6 \mathrm{GHz}$. Scientific applications with the SKA using such a group of sources applied to studies of the nearby universe, including the Milky Way galaxy and the Local Group of galaxies, are described in Imai et al. (2016). Although that case-study report discussed the scientific applications conservatively based on only "in-beam" PR astrometry, our results indicate that MV would provide further benefits.

Pulsars at $1.6 \mathrm{GHz}$. Our empirically estimated MV astrometric accuracy of $\sim 100 \mu$ as at $1.6 \mathrm{GHz}$ with VLBA observations, using calibrators more than $\sim 2^{\circ}$ away, is the state of the art, only comparable with "in-beam" PR observations with a calibrator of $\sim 10 \mathrm{~s}$ arcminutes away (Deller et al. 2013, 2016). This improvement is expected to continue to apply at all angular scales. Hence, using three calibrators within the SKAMid antenna beams and MV will result in a further increase by one order of magnitude of the astrometric precision, extrapolating from our comparative study. In general, allowing for larger angular separations makes it possible to select good calibrator sources, which are fundamental for multi-epoch studies. With the higher probabilities of finding suitable calibrators, the general applicability is also highly increased. 
In some cases, such as pulsar observations in the galactic plane, it might even be desirable to use calibrators out of the plane to reduce the effect of scattering. This would be possible using MV.

Methanol Masers at $6.7 \mathrm{GHz}$. High precision astrometry observations of methanol masers at $6.7 \mathrm{GHz}$ holds the prospect to contribute to the successful program of 3D mapping of our Galaxy, as a complement to the precise water maser measurements (Reid et al. 2014). However, the advanced PR strategies used at $22 \mathrm{GHz}$ in the tropospheric-dominated regime fail to provide high precision astrometry at $6.7 \mathrm{GHz}$, as in the ionospheric-dominated regime. Therefore, MV with fast source switching between sources, or simultaneous observations if possible, provides a strategy for superior calibration of the tropospheric and ionospheric errors, resulting in precise astrometry.

The VLBA is operated by National Radio Astronomy Observatory and is a facility of the National Science Foundation operated under cooperative agreement with Associated Universities Inc. G.O. and H.I. have been supported by the JSPS Bilateral Collaboration Program and KAKENHI programs 25610043 and 16H02167. M.R., R.D., G.O., and H.I. acknowledge support from DFAT grant AJF-124. G.O. was also supported by a MEXT scholarship from the Japanese Government. This research has made use of the SIMBAD database, operated at CDS, Strasbourg, France.

\section{References}

Alef, W. 1988, in IAU Symp. 129, The Impact of VLBI on Astrophysics and Geophysics, ed. M. J. Reid \& J. M. Moran (Cambridge, MA: Kluwer), 523

Asaki, Y., Sudou, H., Kono, Y., et al. 2007, PASJ, 59, 397

Deller, A. T., Boyles, J., Lorimer, D. R., et al. 2013, ApJ, 770, 145

Deller, A. T., Vigeland, S. J., Kaplan, D. L., et al. 2016, ApJ, 828, 8

Dodson, R., Rioja, M., Asaki, Y., et al. 2013, AJ, 145, 147
Dodson, R., Rioja, M., Molina, S., \& Gómez, J. 2017, ApJ, 834, 177

Doi, A., Fujisawa, K., Habe, A., et al. 2006, PASJ, 58, 777

Fomalont, E. B., Goss, W. M., Beasley, A. J., \& Chatterjee, S. 1999, AJ, 117,3025

Fomalont, E. B., \& Kopeikin, S. 2002, in Proc. 6th EVN Symp., Phase Referencing Using Several Calibrator Sources,, ed. E. Ros et al. (Bonn: Max-Planck-Institut für Radioastronomie), 53

Godfrey, L., Bignall, H., \& Tingay, S. 2011, PASA, 29, 42

GraphPad 2014, Statistics with $\mathrm{n}=2$, http://www.graphpad.com/support/ faqid $/ 591 /$

Greisen, E. W. 2003, AIPS, the VLA, and the VLBA (Dordrecht: Kluwer)

Honma, M., Tamura, Y., \& Reid, M. J. 2008, PASJ, 60, 951

Imai, H., Burns, R. A., Yamada, Y., et al. 2016, arXiv:1603.02042

Jimenez-Monferrer, S., Rioja, M. J., Dodson, R., Smirnov, O., \& Guirado, J. C. 2010, in 10th European VLBI Network Symp. and EVN Users Meeting: VLBI and the New Generation of Radio Arrays, Manchester, Proc. Science 125,84

Marcaide, J. M., \& Shapiro, I. I. 1984, ApJ, 276, 56

Martí-Vidal, I., Marcaide, J. M., Guirado, J. C., Pérez-Torres, M. A., \& Ros, E. 2008, A\&A, 478, 267

Orosz, G., Imai, H., Dodson, R., et al. 2017, AJ, accepted

Paragi, Z., Godfrey, L., Reynolds, C., et al. 2015, arXiv:1412.5971

Porcas, R. W. 2009, A\&A, 505, L1

Porcas, R. W., \& Rioja, M. J. 2002, in Proc. 6th EVN Symp., VLBI phasereference investigations at $86 \mathrm{GHz}$, ed. E. Ros et al. (Bonn: Max-PlanckInstitut für Radioastronomie), 65

Reid, M. J., \& Brunthaler, A. 2004, ApJ, 616, 872

Reid, M. J., \& Honma, M. 2014, ARA\&A, 52, 339

Reid, M. J., Menten, K. M., Brunthaler, A., et al. 2014, ApJ, 783, 130

Rioja, M., \& Dodson, R. 2011, AJ, 141, 114

Rioja, M., Dodson, R., Porcas, R. W., et al. 2009, in 8th Int. e-VLBI Workshop Revisited "Cluster-Cluster" VLBI with future multi-beam low frequency radio interferometers (Trieste: SISSA), 14

Rioja, M. J., \& Porcas, R. W. 2000, A\&A, 355, 552

Rioja, M. J., Porcas, R. W., Desmurs, J.-F., et al. 2002, in Proc. 6th EVN Symp., VLBI observations in Cluster-Cluster mode at $1.6 \mathrm{GHz}$, ed. E. Ros et al. (Bonn: Max-Planck-Institut für Radioastronomie), 57

Rioja, M. J., Stevens, E., Gurvits, L., et al. 1997, VA, 41, 213

Shepherd, M. C., Pearson, T. J., \& Taylor, G. B. 1994, BAAS, 26, 987

Wrobel, J., Walker, R., Benson, J., \& A. B. 2000, VLBA Scientific Memorandum n. 24: Strategies for Phase Referencing with the VLBA, Tech. Rep., 24 NRAO 